\title{
Underpowered or negative? A crucial distinction
}

\author{
Ole L. Dollerup ${ }^{1,2} \cdot$ Jonas T. Treebak ${ }^{1} \cdot$ Niels Jessen $^{3,4}$ (D)
}

Received: 27 February 2019 / Accepted: 1 March 2019 / Published online: 25 March 2019

(C) Springer-Verlag GmbH Germany, part of Springer Nature 2019

Keywords Clinical trial $\cdot$ Human $\cdot$ Hyperinsulinaemic-euglycaemic clamp $\cdot$ Insulin sensitivity $\cdot \mathrm{NAD}^{+}$metabolism . Nicotinamide riboside

\section{Abbreviation}

HEC Hyperinsulinaemic-euglycaemic clamp

To the Editor: Schrauwen and colleagues have recently reviewed the emerging field of $\mathrm{NAD}^{+}$metabolism in Diabetologia [1]. Their manuscript includes a description of a clinical trial of dietary nicotinamide riboside supplementation effects on whole body insulin sensitivity in obese men, conducted by us, together with our colleagues at Aarhus and Copenhagen Universities, Denmark [2]. As posted on clinicaltrials.gov (NCT02303483), this placebo-controlled, randomised clinical trial used $M$ value determined by the hyperinsulinaemic-euglycaemic clamp (HEC) technique, the gold-standard approach for directly quantifying insulin sensitivity, as the primary endpoint.

We report a negative outcome of the trial, and while this may be disappointing in terms of the possible future use of nicotinamide riboside supplementation for the prevention of type 2 diabetes, we believe it is important to publish such outcomes and to cite these correctly. We were therefore

\section{Niels Jessen}

Niels.Jessen@biomed.au.dk

1 Novo Nordisk Foundation Center for Basic Metabolic Research, Section for Integrative Physiology, Faculty of Health and Medical Sciences, University of Copenhagen, Copenhagen, Denmark

2 Medical Research Laboratory, Department of Clinical Medicine, Aarhus University, Aarhus, Denmark

3 Steno Diabetes Center Aarhus (SDCA), Aarhus University Hospital and Central Denmark Region, Hedeager 3, DK-8200 Aarhus $\mathrm{N}$, Denmark

4 Department of Biomedicine, Aarhus University, Aarhus, Denmark disconcerted to find the following description of our manuscript in the review by Schrauwen and colleagues: 'The authors concluded that this study was underpowered and future studies should be larger and focus on other variables of metabolic health'. We find this to be a severe misrepresentation of a central conclusion of our work.

As outlined in our manuscript, power calculations were conducted prior to enrolment into our study to determine an appropriate sample size for detecting clinically meaningful differences in the primary endpoint and to try to evade type I and type II errors resulting from insufficient sample size. We powered the study to detect a difference in the HEC-derived $M$ value of $1.5 \mathrm{mg} \mathrm{kg}^{-1} \mathrm{~min}^{-1}$, which has previously been achieved with insulin-sensitising drugs or exercise [3, 4]. We used a conservative estimate of the SD of $1.6 \mathrm{mg} \mathrm{kg}^{-1} \mathrm{~min}^{-1}$ in the power calculation, based on in-house experience from previous clamp examinations [2]. The primary endpoint, the $M$ value, was not affected by nicotinamide riboside supplementation ( $p$ for interaction $=0.68$ ). However, the actual SD for the difference between the baseline and the end-of-study $M$ value within the placebo group was only $0.48 \mathrm{mg} \mathrm{kg}^{-1} \mathrm{~min}^{-1}$. Therefore, in retrospect, the assumed SD was probably too conservative. With an SD of 0.5 and $n$ of 40 , we would be able to detect a significant difference in the $M$ value of $0.45 \mathrm{mg} \mathrm{kg}^{-1} \mathrm{~min}^{-1}$ with a power of 0.80 . Hence, we do not suspect that the lack of effects of nicotinamide riboside can be attributed to the study being underpowered (i.e. a type II error). In addition to the $M$ value, our study included several predefined secondary endpoints, and the combined outcomes of these were also negative. In line with standard study design, the trial was powered to detect significant differences in the primary endpoint and, therefore, where the risk of a type II error was discussed in our paper, this was in terms of the secondary endpoints [2].

When citing a clinical trial, it is important to respect the distinction between endpoints. When quoting the authors as 
concluding that the study was underpowered, unless otherwise stated, it will be assumed that this conclusion refers to the primary outcome of the study. Dietary supplements, such as nicotinamide riboside, are already commercially available and are being promoted as facilitating healthy ageing in humans by an industry that is not subject to the same strict regulatory demands as the traditional pharmaceutical industry. This stresses the need for scientific testing of safety and efficacy of dietary supplements in rigorously designed human clinical trials, and the outcomes of such trials should be reported correctly in subsequent review papers, irrespective of whether the outcome is positive or negative.

Duality of interest The authors declare that there is no duality of interest associated with this manuscript.

Contribution statement All authors were responsible for drafting the article and revising it critically for important intellectual content. All authors approved the version to be published.

\section{References}

1. Connell NJ, Houtkooper RH, Schrauwen P (2019) NAD ${ }^{+}$metabolism as a target for metabolic health: have we found the silver bullet? Diabetologia. https://doi.org/10.1007/s00125-019-4831-3

2. Dollerup OL, Christensen B, Svart M et al (2018) A randomized placebo-controlled clinical trial of nicotinamide riboside in obese men: safety, insulin-sensitivity, and lipid-mobilizing effects. Am J Clin Nutr 108(2):343-353. https://doi.org/10.1093/ajcn/nqy132

3. Inzucchi SE, Maggs DG, Spollett GR et al (1998) Efficacy and metabolic effects of metformin and troglitazone in type II diabetes mellitus. N Engl J Med 338(13):867-872. https://doi.org/10.1056/ NEJM199803263381303

4. Malin SK, Haus JM, Solomon TPJ et al (2013) Insulin sensitivity and metabolic flexibility following exercise training among different obese insulin-resistant phenotypes. Am J Physiol Endocrinol Metab 305(10):E1292-E1298. https://doi.org/10.1152/ajpendo. 00441.2013

Publisher's note Springer Nature remains neutral with regard to jurisdictional claims in published maps and institutional affiliations. 